\title{
Fermionic representations of integrable lattice systems
}

\author{
Frank Göhmann 调 and Shuichi Murakami部 \\ †Institute for Theoretical Physics, \\ State University of New York at Stony Brook, \\ Stony Brook, NY 11794-3840, USA \\ $\ddagger$ Department of Applied Physics, University of Tokyo, \\ Hongo 7-3-1, Bunkyo-ku, Tokyo 113, Japan
}

\begin{abstract}
We develop a general scheme for the use of Fermi operators within the framework of integrable systems. This enables us to read off a fermionic Hamiltonian from a given solution of the YangBaxter equation and to express the corresponding $L$-matrix and the generators of symmetries in terms of Fermi operators. We illustrate our approach through a number of examples. Our main example is the algebraic Bethe ansatz solution of the Hubbard model in the infinite coupling limit.
\end{abstract}

*e-mail: goehmann@insti.physics.sunysb.edu

§e-mail: murakami@appi.t.u-tokyo.ac.jp 


\section{Introduction}

The purpose of this article is to consider graded vector spaces and the graded Yang-Baxter algebra in such a way that the explicit construction of integrable models in terms of Fermi operators becomes easy. We will present simple general formulae, which will enable expression of Hamiltonian, $L$ matrix, Yang-Baxter algebra and generators of symmetries in terms of Fermi operators, once a solution of the Yang-Baxter equation is given.

The material developed below has its origin in two articles of Kulish and Sklyanin [1, 2], where fundamental, graded integrable lattice systems were considered for the first time. Although most of the basic ideas are outlined in [1], 2], these articles remain rather sketchy as far as concrete representations in terms of Fermi operators are concerned. We shall try to explain in the following that this is a topic of its own interest.

We would like to emphasize that the construction of fermionic representations is a subject which has to be seen separately from the construction of integrable models invariant under Lie superalgebras or their deformations (cf e.g. [3, [, 5]). Although Lie superalgebra invariant models are most naturally represented in terms of Fermi operators, there is no need to do so. On the other hand, there are models, such as the Hubbard model, or its infinite coupling limit, to be treated below, which are not invariant under graded algebras, but have their most interesting interpretation in terms of Fermi operators. The possibility of connecting a given solution of the Yang-Baxter equation with a fermionic representation is only restricted by a rather weak compatibility condition (eq. (26)). Thus there may be different fermionic representations of the same model, which correspond to different gradings.

One possibility to connect spin models with fermionic models, which is frequently encountered in the literature, is the Jordan-Wigner transformation. This transformation is mostly applied on the level of Hamiltonians. Applying it to the Yang-Baxter algebra is a feasible, yet cumbersome task. The Jordan-Wigner transformation led to important progress, e.g. in the understanding of the Hubbard model [6, 0]. We shall argue however that it is an unnecessary element for the construction of fermionic representations and that the route taken in the present article leads to a clearer understanding of the issue. The Jordan-Wigner transformation does not preserve the boundary conditions. It may obstruct symmetries, and is not easily generalizable to an arbitrary number of internal degrees of freedom. 
The plan of the present paper is as follows. We first present some necessary mathematical preliminaries. We introduce the parity, a concept of odd and even on the basis of a finite dimensional vector space, which is then called a graded vector space. Later this will allows us to make contact with Fermi operators. We extend the concept of parity to endomorphisms of the vector space and to tensor powers of endomorphisms. The central definition is equation (12), which provides an embedding of a "local" basis $\left\{e_{\alpha}^{\beta}\right\}$ of endomorphisms into a "chain of $L$ sites", $\left\{e_{\alpha}^{\beta}\right\} \rightarrow\left\{e_{j_{\alpha}}^{\beta}\right\}$, in such a way that the $e_{j_{\alpha}}^{\beta}$, depending on the value of $\alpha$ and $\beta$, either commute or anticommute. The $e_{j_{\alpha}}^{\beta}$ are "graded analogs of spin operators". It will turn out later that they can be directly replaced by fermionic projection operators. This is an advantage of considering the grading on a basis rather than on coordinates. Before turning to fermions, we have to develop the quantum inverse scattering method using the basis $\left\{e_{j_{\alpha}}^{\beta}\right\}$. We try to keep the analogy to the non-graded case as close as possible and try to emphasize in our presentation the modifications due to the grading. We discuss the general case of global symmetries, which, due to the grading are subalgebras of Lie superalgebras. Later, after having introduced Fermi operators, we look at the example of gauge transformations, where we also discuss the local case.

Finally, we illustrate our approach with a number of examples. Most of these examples will be familiar to the reader. They were chosen for this reason. The last example, however, is new and of its own interest. Our approach allows us to identify the $R$-matrix of the Hubbard model in the infinite coupling limit as a member of a family of $R$-matrices of $\operatorname{su}(\mathrm{N}) \mathrm{XX}$ chains, which was recently proposed by Maassarani and Mathieu [8]. Since the example is new, we work it out in some detail. We perform an algebraic Bethe ansatz for the model and investigate its symmetries and the question of completeness of the algebraic Bethe ansatz eigenstates.

\section{Graded vector spaces}

Within the formalism of second quantization a physical lattice system can be entirely described by a set of creation and annihilation operators $a_{j \alpha}^{+}, a_{j \alpha}$ of particles at site $j$ of the lattice. Depending on the index $\alpha$ these particles may be bosons or fermions. Accordingly, they commute or anticommute at different sites,

$$
a_{j \alpha} a_{k \beta} \pm a_{k \beta} a_{j \alpha}=0 \quad, \quad j \neq k
$$


Let us consider systems with a finite number of states per site, say $m$ bosonic and $n$ fermionic states, $\alpha=1, \ldots, m+n$. We can look for matrix representations of the operators $a_{j \alpha}$ acting on tensor products of the local space $\mathbf{C}^{m+n}$. For spinless fermions, for instance, such a representation is provided by the Jordan-Wigner transformation. Unoccupied sites are bosonic, occupied sites are fermionic.

If we want to describe the general situation, it turns out to be useful to reverse the above reasoning. Let us start from a local space of states $V$, which is then isomorphic to $\mathbf{C}^{m+n}$, and let us impose an additional structure, the parity, from the outset. Let $V=V_{0} \oplus V_{1}$, and call $v_{0} \in V_{0}$ even, $v_{1} \in V_{1}$ odd. The subspaces $V_{0}$ and $V_{1}$ are called the homogeneous components of $V$. The parity $p$ is a function $V_{i} \longrightarrow Z_{2}$ defined on the homogeneous components of $V$,

$$
p\left(v_{i}\right)=i \quad, \quad i=0,1 \quad, \quad v_{i} \in V_{i} .
$$

The vector space $V$ equipped with this structure is called a graded vector space or superspace. Fix a basis $\left\{e_{1}, \ldots, e_{m+n}\right\}$ of definite parity. Let $p(\alpha):=p\left(e_{\alpha}\right)$. Since we want to construct an algebra of commuting and anticommuting operators, we have to extend the concept of parity to operators in $\operatorname{End}(V)$ and to tensor products of these operators. Let $e_{\alpha}^{\beta} \in \operatorname{End}(V)$, $e_{\alpha}^{\beta} e_{\gamma}=\delta_{\gamma}^{\beta} e_{\alpha} .\left\{e_{\alpha}^{\beta}\right\}$ is a basis of $\operatorname{End}(V)$. If we represent $e_{\gamma}$ as a column matrix with only non-vanishing entry 1 in row $\gamma$, then $e_{\alpha}^{\beta}$ is an $(m+n) \times(m+n)$ matrix with only non-vanishing entry 1 in row $\alpha$ and column $\beta$. Define the parity of rows and columns,

$$
p_{r}\left(e_{\alpha}^{\beta}\right)=p(\alpha) \quad, \quad p_{c}\left(e_{\alpha}^{\beta}\right)=p(\beta)
$$

and the parity of matrices

$$
p\left(e_{\alpha}^{\beta}\right)=p(\alpha)+p(\beta) .
$$

With this definition $\operatorname{End}(V)$ becomes a graded vector space.

Take $X, Y$ from the homogeneous components of $\operatorname{End}(V)$, and define the superbracket

$$
[X, Y]_{ \pm}=X Y-(-1)^{p(X) p(Y)} Y X
$$

Extend the superbracket linearly in both of its arguments to $\operatorname{End}(V)$. Then, $\operatorname{End}(V)$ endowed with the superbracket becomes the Lie superalgebra $\operatorname{gl}(m \mid n)$. Note that the above definition of a superbracket makes sense in 
any graded algebra. We will also use it in the context of graded tensor powers of $\operatorname{End}(V)$, which is our next issue.

The notion of a grading may be extended to the $L$-fold tensor product of $\operatorname{End}(V)$, setting

$$
\begin{aligned}
p_{r}\left(e_{\alpha_{1}}^{\beta_{1}} \otimes \ldots \otimes e_{\alpha_{L}}^{\beta_{L}}\right) & =p\left(\alpha_{1}\right)+\ldots+p\left(\alpha_{L}\right), \\
p_{c}\left(e_{\alpha_{1}}^{\beta_{1}} \otimes \ldots \otimes e_{\alpha_{L}}^{\beta_{L}}\right) & =p\left(\beta_{1}\right)+\ldots+p\left(\beta_{L}\right), \\
p\left(e_{\alpha_{1}}^{\beta_{1}} \otimes \ldots \otimes e_{\alpha_{L}}^{\beta_{L}}\right) & =p\left(\alpha_{1}\right)+p\left(\beta_{1}\right)+\ldots+p\left(\alpha_{L}\right)+p\left(\beta_{L}\right) .
\end{aligned}
$$

It can be seen from the last formula, that homogeneous elements $A=$ $A_{\beta_{1} \ldots \beta_{L}}^{\alpha_{1} \ldots \alpha_{L}} e_{\alpha_{1}}^{\beta_{1}} \otimes \ldots \otimes e_{\alpha_{L}}^{\beta_{L}}$ of $(\operatorname{End}(V))^{\otimes L}$ with parity $p(A)$ are characterized by the equation

$$
(-1)^{\sum_{j=1}^{L}\left(p\left(\alpha_{j}\right)+p\left(\beta_{j}\right)\right)} A_{\beta_{1} \ldots \beta_{L}}^{\alpha_{1} \ldots \alpha_{L}}=(-1)^{p(A)} A_{\beta_{1} \ldots \beta_{L}}^{\alpha_{1} \ldots \alpha_{L}} .
$$

This implies that $A B$ is homogeneous with parity

$$
p(A B)=p(A)+p(B) \quad,
$$

if $A$ and $B$ are homogeneous.

The above definitions allow us to introduce another tensor product structure $\otimes_{s}$, which is called the graded or supertensor product, on exterior powers of $\operatorname{End}(V)$. Choose $v$ and $w$ from the homogeneous components of $(\operatorname{End}(V))^{\otimes k}$ and $(\operatorname{End}(V))^{\otimes l}$, respectively. Then, by definition,

$$
v \otimes_{s} w=(-1)^{p(v) p_{r}(w)} v \otimes w .
$$

As a simple consequence of this definition, the supertensor product is associative, $\left(u \otimes_{s} v\right) \otimes_{s} w=u \otimes_{s}\left(v \otimes_{s} w\right)$. This follows first for $u, v, w$ taken from the homogeneous components of certain powers of $\operatorname{End}(V)$, and then by linearity for arbitrary $u, v$ and $w$.

Here comes the central definition of the paper. The supertensor product induces an embedding of $e_{\alpha}^{\beta}$ into $(\operatorname{End}(V))^{\otimes L}$,

$$
\begin{aligned}
& e_{j_{\alpha}}^{\beta}=I_{m+n}^{\otimes_{s}(j-1)} \otimes_{s} e_{\alpha}^{\beta} \otimes_{s} I_{m+n}^{\otimes_{s}(L-j)} \\
& =(-1)^{(p(\alpha)+p(\beta)) \sum_{k=j+1}^{L} p\left(\gamma_{k}\right)} I_{m+n}^{\otimes(j-1)} \otimes e_{\alpha}^{\beta} \otimes e_{\gamma_{j+1}}^{\gamma_{j+1}} \otimes \ldots \otimes e_{\gamma_{L}}^{\gamma_{L}}
\end{aligned}
$$


$I_{m+n}$ in this equation denotes the $(m+n) \times(m+n)$ unit matrix. In the second equation summation over double tensor indices is understood. The index $j$ on the left hand side of (12) will be called site index. The matrices $e_{j}^{\beta}$ realize relations of the form (1). For $j \neq k$ we find

$$
e_{j}^{\beta} e_{k_{\gamma}}^{\delta}=(-1)^{(p(\alpha)+p(\beta))(p(\gamma)+p(\delta))} e_{k_{\gamma}}^{\delta} e_{j_{\alpha}}^{\beta} .
$$

It follows from eq. (13) that $e_{j_{\alpha}}^{\beta}$ is homogeneous, and that

$$
p\left(e_{j_{\alpha}}^{\beta}\right)=p(\alpha)+p(\beta) .
$$

Hence, in agreement with intuition, eq. (14) says that odd matrices mutually anticommute, whereas even matrices commute with each other as well as with the odd matrices. For products of matrices $e_{j_{\alpha}}^{\beta}$ which are acting on the same site (13) implies

$$
e_{j_{\alpha}}^{\beta} e_{j_{\gamma}}^{\delta}=\delta_{\gamma}^{\beta} e_{j_{\alpha}}^{\delta}
$$

Using the superbracket, (14) and (16) may be combined to

$$
\left[e_{j_{\alpha}}^{\beta}, e_{k_{\gamma}}^{\delta}\right]_{ \pm}=\delta_{j k}\left(\delta_{\gamma}^{\beta} e_{j_{\alpha}}^{\delta}-(-1)^{(p(\alpha)+p(\beta))(p(\gamma)+p(\delta))} \delta_{\alpha}^{\delta} e_{j_{\gamma}}^{\beta}\right)
$$

The right hand side of the latter equation with $j=k$ gives the structure constants of the Lie superalgebra $\operatorname{gl}(m \mid n)$ with respect to the basis $\left\{e_{j_{\alpha}}^{\beta}\right\}$.

\section{The permutation operator}

The permutation operator plays an important role in the construction of local integrable lattice models. In the graded case it requires the following modifications of signs,

$$
P_{j k}=(-1)^{p(\beta)} e_{j}^{\beta} e_{k \beta}^{\alpha}
$$

This operator induces the action of the symmetric group $S^{L}$ on the site indices of the matrices $e_{j}^{\beta}$. The following properties are easily verified, they follow from (14) and (16),

$$
\begin{aligned}
P_{k j} & =P_{j k}, \\
P_{j j} & =(m-n) \mathrm{id}, \\
P_{j k}^{2} & =\mathrm{id}, \quad j \neq k, \\
P_{j k} e_{k_{\alpha}}^{\beta} & =e_{j \alpha}^{\beta} P_{j k}, \\
P_{j k} e_{l \alpha}^{\beta} & =e_{l \alpha}^{\beta} P_{j k}, \quad j \neq l \neq k .
\end{aligned}
$$




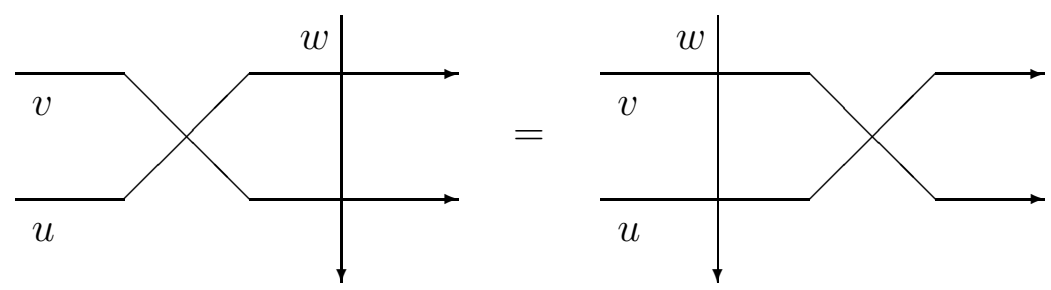

Figure 1: The Yang-Baxter equation is most easily memorized in graphical form

Because of $(d)$ and $(e) P_{j k}$ generates a faithful representation of $S^{L}$,

$$
P_{j k} P_{k l}=P_{j l} P_{j k}=P_{k l} P_{j l}
$$

Let $L=2$. Then

$$
P_{12}=(-1)^{p(\beta)} e_{1 \alpha}^{\beta} e_{2 \beta}^{\alpha}=(-1)^{p(\alpha) p(\beta)} e_{\alpha}^{\beta} \otimes e_{\beta}^{\alpha}=(-1)^{p(\alpha) p(\beta)} \delta_{\delta}^{\alpha} \delta_{\gamma}^{\beta} e_{\alpha}^{\gamma} \otimes e_{\beta}^{\delta}
$$

From the right-hand side of this equation we can read off the matrix elements of $P_{12}$ with respect to the canonical basis of $\operatorname{End}(V \otimes V)$.

\section{The graded Yang-Baxter algebra}

In the present context it is most suitable to interpret the Yang-Baxter equation as a set of functional equations for the matrix elements of an $(m+n)^{2} \times$ $(m+n)^{2}$-matrix $R(u, v)$. We may represent it in graphical form as shown in Figure 1, where each vertex corresponds to a factor in the equation

$$
R_{\alpha^{\prime} \beta^{\prime}}^{\alpha \beta}(u, v) R_{\alpha^{\prime \prime} \gamma^{\prime}}^{\alpha^{\prime} \gamma}(u, w) R_{\beta^{\prime \prime} \gamma^{\prime \prime}}^{\beta^{\prime} \gamma^{\prime}}(v, w)=R_{\beta^{\prime} \gamma^{\prime}}^{\beta \gamma}(v, w) R_{\alpha^{\prime} \gamma^{\prime \prime}}^{\alpha \gamma^{\prime}}(u, w) R_{\alpha^{\prime \prime} \beta^{\prime \prime}}^{\alpha^{\prime} \beta^{\prime}}(u, v) .
$$

Note that there is a direction assigned to every line in figure 1, which is indicated by the tips of the arrows. Therefore every vertex has an orientation, and vertices and $R$-matrices can be identified according to figure 2 , where indices have been supplied to a vertex. Summation is over all inner lines in figure 1. 


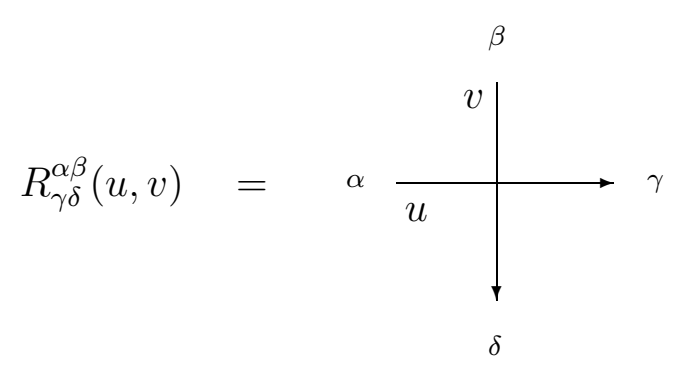

Figure 2: Identification of the $R$-matrix with a vertex

Starting from the Yang-Baxter equation we will construct a fundamental graded representation of the Yang-Baxter algebra. For comparison let us briefly recall the non-graded case $(n=0)$. Define $\check{R}(u, v)$ as

$$
\check{R}_{\gamma \delta}^{\alpha \beta}(u, v)=R_{\gamma \delta}^{\beta \alpha}(u, v)
$$

Introduce the $L$-matrix at site $j$,

$$
L_{j \beta}^{\alpha}(u, v)=R_{\beta \delta}^{\alpha \gamma}(u, v) e_{j \gamma}^{\delta}
$$

Then multiplication of the Yang-Baxter equation (22) by $e_{j}^{\gamma_{\gamma}^{\prime \prime}}$ implies that

$$
\check{R}(u, v)\left(L_{j}(u, w) \otimes L_{j}(v, w)\right)=\left(L_{j}(v, w) \otimes L_{j}(u, w)\right) \check{R}(u, v)
$$

where the tensor product is now a tensor product between matrices, according to the convention $(A \otimes B)_{\beta \delta}^{\alpha \gamma}=A_{\beta}^{\alpha} B_{\delta}^{\gamma}$. We may replace $L_{j}$ in (25) by some matrix $T$ and may interpret it as defining an abstract algebra for the matrix elements of $T$. This algebra is called a Yang-Baxter algebra with $R$-matrix $R$. $L_{j}$ is called its fundamental representation. Since $L$-matrices at different sites commute, any product of $L$-matrices with different site indices is another representation of the same Yang-Baxter algebra.

The construction of a graded Yang-Baxter algebra and its fundamental representation requires only minimal modifications of the above scheme. Let us assume we are given a solution of (22), which is compatible with the grading in the sense that

$$
R_{\gamma \delta}^{\alpha \beta}(u, v)=(-1)^{p(\alpha)+p(\beta)+p(\gamma)+p(\delta)} R_{\gamma \delta}^{\alpha \beta}(u, v)
$$


Define a graded $L$-matrix at site $j$ as

$$
\mathcal{L}_{j \beta}^{\alpha}(u, v)=(-1)^{p(\alpha) p(\gamma)} R_{\beta \delta}^{\alpha \gamma}(u, v) e_{j_{\gamma}^{\delta}}^{\delta}
$$

Eq. (26) implies that the matrix elements of $\mathcal{L}_{j}(u, v)$ are of definite parity,

$$
p\left(\mathcal{L}_{j}^{\alpha}(u, v)\right)=p(\alpha)+p(\beta) \quad,
$$

and that they commute as

$$
\mathcal{L}_{j \beta}^{\alpha}(u, v) \mathcal{L}_{k \delta}^{\gamma}(w, z)=(-1)^{(p(\alpha)+p(\beta))(p(\gamma)+p(\delta))} \mathcal{L}_{k \delta}^{\gamma}(w, z) \mathcal{L}_{j \beta}^{\alpha}(u, v)
$$

It further follows from the Yang-Baxter equation (22) and from eq. (26) that

$$
\check{R}(u, v)\left(\mathcal{L}_{j}(u, w) \otimes_{s} \mathcal{L}_{j}(v, w)\right)=\left(\mathcal{L}_{j}(v, w) \otimes_{s} \mathcal{L}_{j}(u, w)\right) \check{R}(u, v)
$$

In analogy to the non-graded case above, the supertensor product in this equation is to be understood as a supertensor product of matrices with noncommuting entries, $\left(A \otimes_{s} B\right)_{\beta \delta}^{\alpha \gamma}=(-1)^{(p(\alpha)+p(\beta)) p(\gamma)} A_{\beta}^{\alpha} B_{\delta}^{\gamma}$. In a sense this definition is a contravariant counterpart of equation (11). Given matrices $A$, $B, C, D$ with operator valued entries, which mutually commute according to the same rule as $\mathcal{L}_{j}$ and $\mathcal{L}_{k}$ in eq. (29), we obtain for the product of two supertensor products

$$
\left(A \otimes_{s} B\right)\left(C \otimes_{s} D\right)=A C \otimes_{s} B D
$$

Eq. (30) may be interpreted as defining a graded Yang-Baxter algebra with $R$-matrix $R . \mathcal{L}_{j}$ is then its fundamental representation.

Starting from (30) we can construct integrable lattice models as in the non-graded case. Let us briefly recall the construction with emphasis on the modifications that appear due to the grading. Define a monodromy matrix $\mathcal{T}(u, v)$ as an $L$-fold ordered product of fundamental $L$-matrices,

$$
\mathcal{T}(u, v)=\mathcal{L}_{L}(u, v) \ldots \mathcal{L}_{1}(u, v)
$$

Due to eq. (10) the matrix elements of $\mathcal{T}(u, v)$ are homogeneous with parity $p\left(\mathcal{T}_{\beta}^{\alpha}(u, v)\right)=p(\alpha)+p(\beta)$. Repeated application of (30) and (31) shows that this monodromy matrix is a representation of the graded Yang-Baxter algebra,

$$
\check{R}(u, v)\left(\mathcal{T}(u, w) \otimes_{s} \mathcal{T}(v, w)\right)=\left(\mathcal{T}(v, w) \otimes_{s} \mathcal{T}(u, w)\right) \check{R}(u, v)
$$


In the non-graded case $(n=0)$ the supertensor product in (33) agrees with the usual tensor product. Multiplying (33) by $\check{R}^{-1}(u, v)$ and taking the trace of the whole equation then implies that $[\operatorname{tr}(\mathcal{T}(u, w)), \operatorname{tr}(\mathcal{T}(v, w))]=0$, and the transfer matrix $\operatorname{tr}(\mathcal{T}(u, w))$ provides a generating function of mutually commuting operators, which may take the role of conserved quantities of an integrable lattice model. For non-trivial grading the trace has to be replaced by the supertrace, which is generally defined as $\operatorname{str}(A)=$ $(-1)^{\sum_{j=1}^{N} p\left(\alpha_{j}\right)} A_{\alpha_{1} \ldots \alpha_{N}}^{\alpha_{1} \ldots \alpha_{N}}$. Then (33) implies that

$$
[\operatorname{str}(\mathcal{T}(u, w)), \operatorname{str}(\mathcal{T}(v, w))]=0,
$$

in complete analogy with the non-graded case.

Let us assume that $R(u, v)$ is a regular solution of the Yang-Baxter equation. This means that there are values $u_{0}, v_{0}$ of the spectral parameters such that $R_{\gamma \delta}^{\alpha \beta}\left(u_{0}, v_{0}\right)=\delta_{\delta}^{\alpha} \delta_{\gamma}^{\beta}$. Then (27) implies

$$
\mathcal{L}_{j}^{\alpha}\left(u_{0}, v_{0}\right)=(-1)^{p(\alpha) p(\beta)} e_{j}^{\alpha} \quad,
$$

and we can easily see that the supertrace of the monodromy matrix at $\left(u_{0}, v_{0}\right)$ generates a shift by one site,

$$
\begin{aligned}
\operatorname{str}\left(\mathcal{T}\left(u_{0}, v_{0}\right)\right) & =(-1)^{p(\alpha)} \mathcal{T}_{\alpha}^{\alpha}\left(u_{0}, v_{0}\right) \\
& =(-1)^{\sum_{k=1}^{L-1} p\left(\beta_{k}\right)} e_{1}^{\beta_{1}} e_{2} e_{\beta_{1}}^{\beta_{2}} e_{3} \beta_{\beta_{2}}^{\beta_{3}} \ldots e_{L}^{\beta_{\beta_{L-1}}} \\
& =P_{12} P_{23} \ldots P_{L-1 L}=: \hat{U} .
\end{aligned}
$$

This implies that $\tau(u):=\ln \left(\operatorname{str}\left(\mathcal{T}\left(u, v_{0}\right)\right)\right)$ generates a sequence of local operators [9] which, as a consequence of (34), mutually commute,

$$
\tau(u)=\mathrm{i} \hat{\Pi}+\left(u-u_{0}\right) \hat{H}+\mathcal{O}\left(\left(u-u_{0}\right)^{2}\right) \quad .
$$

$\hat{\Pi}$ in this expansion is the momentum operator. On a lattice, where the minimal possible shift is by one site, and thus $\hat{U}$ rather than $\hat{\Pi}$ is the fundamental geometrical operator, some care is required in the definition of $\hat{\Pi}$. As was shown in [10] a proper definition may be obtained by setting $\Pi:=-\mathrm{i} \ln (\hat{U}) \bmod 2 \pi$ and expressing the function $f(x)=x \bmod 2 \pi$ by its Fourier sum. Then $\hat{\Pi}$ becomes a polynomial in $\hat{U}$.

$$
\hat{\Pi}=\phi \sum_{m=1}^{L-1}\left(\frac{1}{2}+\frac{\hat{U}^{m}}{e^{-i \phi m}-1}\right),
$$


where $\phi=2 \pi / L$. The first order term $\hat{H}$ in the expansion (37) may be interpreted as Hamiltonian. Using (36) it is obtained as

$$
\hat{H}=\sum_{j=1}^{L} H_{j j+1}
$$

where $H_{L L+1}=H_{L 1}$ and

$$
H_{j j+1}=\left.(-1)^{p(\gamma)(p(\alpha)+p(\gamma))} \partial_{u} \check{R}_{\gamma \delta}^{\alpha \beta}\left(u, v_{0}\right)\right|_{u=u_{0}} e_{j}^{\gamma} e_{j+1}^{\delta}
$$

We would like to draw the reader's attention to the following points. (i) The $R$-matrix $\check{R}$ in equation (30) does not undergo a modification due to the grading. (ii) The only necessary compatibility condition which has to be satisfied in order to introduce graded $L$-matrices is equation (26). As we will see in the examples below, this is a weak condition. A given $R$-matrix may be compatible with different gradings, leading to different graded $L$-matrices.

\section{A first example}

As mentioned above the matrices $e_{j}^{\beta}$ for fixed $j$ form "local" representations of $\operatorname{gl}(m \mid n)$. After summing over all sites, we obtain a "global" representation,

$$
\begin{aligned}
E_{\alpha}^{\beta} & =\sum_{j=1}^{L} e_{j_{\alpha}}^{\beta}, \\
{\left[E_{\alpha}^{\beta}, E_{\gamma}^{\delta}\right]_{ \pm} } & =\delta_{\gamma}^{\beta} E_{\alpha}^{\delta}-(-1)^{(p(\alpha)+p(\beta))(p(\gamma)+p(\delta))} \delta_{\alpha}^{\delta} E_{\gamma}^{\beta} .
\end{aligned}
$$

The $E_{\alpha}^{\beta}$ are symmetric in the site indices by construction. Thus $\left[P_{j j+1}, E_{\alpha}^{\beta}\right]=$ 0 , and we obtain a $\operatorname{gl}(m \mid n)$ invariant Hamiltonian, if we are able to find a solution of the Yang-Baxter equation (22), which leads to $H_{j j+1}=P_{j j+1}$ in equation (40). Then, comparing (18) and (40), $\left.\partial_{u} R_{\gamma \delta}^{\alpha \beta}\left(u, v_{0}\right)\right|_{u=u_{0}}=$ $(-1)^{p(\alpha) p(\beta)} \delta_{\gamma}^{\alpha} \delta_{\delta}^{\beta}$. Taking into account regularity, we find the following minimal Ansatz for $R(u, v)$,

$$
R_{\gamma \delta}^{\alpha \beta}(u, v)=\delta_{\delta}^{\alpha} \delta_{\gamma}^{\beta}+(u-v)(-1)^{p(\alpha) p(\beta)} \delta_{\gamma}^{\alpha} \delta_{\delta}^{\beta},
$$

which is indeed a well known rational solution of the Yang-Baxter equation [2]. 


\section{Global symmetries from local symmetries}

We are going to consider now the general case of symmetries of the monodromy matrix, which stem from Lie superalgebra invariance of the $R$-Matrix in a sense to be specified. Choose $x=x_{\beta}^{\alpha} e_{\alpha}^{\beta}$ homogeneous from $g l(m \mid n)$. Let $x_{j}:=x_{\beta}^{\alpha} e_{j}^{\beta}$ and $X:=\sum_{j=1}^{L} x_{j}$. Define $\tilde{R}_{\beta \delta}^{\alpha \gamma}(u, v):=(-1)^{p(\alpha) p(\gamma)} R_{\beta \delta}^{\alpha \gamma}(u, v)$. Assume that $\tilde{R}(u, v)$ satisfies the invariance equation

$$
\begin{aligned}
& \tilde{R}_{\beta^{\prime} \delta}^{\alpha \gamma}(u, v) x_{\beta}^{\beta^{\prime}}+\tilde{R}_{\beta \delta^{\prime}}^{\alpha \gamma}(u, v) x_{\delta}^{\delta^{\prime}} \\
& \quad=(-1)^{p(x)\left(p\left(\alpha^{\prime}\right)+p(\beta)\right)} x_{\alpha^{\prime}}^{\alpha} \tilde{R}_{\beta \delta}^{\alpha^{\prime} \gamma}(u, v)+(-1)^{p(x)(p(\alpha)+p(\beta))} x_{\gamma^{\prime}}^{\gamma} \tilde{R}_{\beta \delta}^{\alpha \gamma^{\prime}}(u, v) .
\end{aligned}
$$

From here we can move step by step to the invariance of the transfer matrix $\operatorname{str}(\mathcal{T}(u, v))$. Contraction of (44) with $e_{j}^{\delta}$ yields

$$
\begin{aligned}
& \mathcal{L}_{j_{\beta^{\prime}}}^{\alpha}(u, v) x_{\beta}^{\beta^{\prime}}+\mathcal{L}_{j}^{\alpha}(u, v) x_{j} \\
& \quad=(-1)^{p(x)\left(p\left(\alpha^{\prime}\right)+p(\beta)\right)} x_{\alpha^{\prime}}^{\alpha} \mathcal{L}_{j \beta}^{\alpha^{\prime}}(u, v)+(-1)^{p(x)(p(\alpha)+p(\beta))} x_{j} \mathcal{L}_{j}^{\alpha}(u, v),
\end{aligned}
$$

and it can be shown by induction over $L$ that the monodromy matrix satisfies

$$
\begin{aligned}
& \mathcal{T}_{\gamma}^{\alpha}(u, v) x_{\beta}^{\gamma}+\mathcal{T}_{\beta}^{\alpha}(u, v) X= \\
& \quad(-1)^{p(x)(p(\gamma)+p(\beta))} x_{\gamma}^{\alpha} \mathcal{T}_{\beta}^{\gamma}(u, v)+(-1)^{p(x)(p(\alpha)+p(\beta))} X \mathcal{T}_{\beta}^{\alpha}(u, v)
\end{aligned}
$$

We finally take the supertrace of this equation and arrive at

$$
[\operatorname{str}(\mathcal{T}(u, v)), X]=0
$$

In many cases the symmetry of the $R$-matrix is evident by construction, e.g. when the $R$-matrix is an intertwiner of representations of quantum groups. Yet there are examples, as Shastry's $R$-matrix of the Hubbard model [6, 7], where the symmetries are less obvious [10, 11]. Moreover, as can be seen by the above derivation, the symmetries of the transfer matrix are determined by the symmetries of $\tilde{R}$ rather than $R$. The symmetries of $\tilde{R}$ depend on the choice of the grading.

It may be argued that, in presence of a grading, the matrix $\tilde{R}$ is more fundamental than $R$, since $\tilde{R}$ determines the $L$-matrix, eq. (27), the symmetries

of the model and, if it exists, the semi-classical limit [1]. Substituting $\tilde{R}$ into 
the Yang-Baxter equation (22), we obtain the so-called graded Yang-Baxter equation, which equivalently might have been taken as the starting point of our section on the graded Yang-Baxter algebra. Since it is the non-graded matrix $\check{R}$, however, which fixes the structure of the Yang-Baxter algebra, eq. (30), we stood away from this point of view.

\section{Representations in terms of fermions}

In this section we shall explain how the various graded objects, which have been introduced so far, can be expressed in terms of Fermi operators. To begin with, consider spinless fermions on a ring of $L$ lattice sites,

$$
\left\{c_{j}, c_{k}\right\}=\left\{c_{j}^{\dagger}, c_{k}^{\dagger}\right\}=0 \quad, \quad\left\{c_{j}, c_{k}^{\dagger}\right\}=\delta_{j k} \quad, \quad j, k=1, \ldots, L \quad .
$$

Locally there are two states, every site is either occupied by a fermion or it is empty. Slightly deviating from the usual language we may say that $c_{j}$ and $c_{j}^{\dagger}$ annihilate or create the occupied state. Let us define a pair $a_{j_{1}}, a_{j 1}^{\dagger}$ of (trivial) annihilation and creation operators of the unoccupied state by setting $a_{j_{1}}=a_{j 1}^{\dagger}=1$, and let us write $a_{j_{2}}=c_{j}, a_{j 2}^{\dagger}=c_{j}^{\dagger}$. Let $n_{j}=c_{j}^{\dagger} c_{j}$ denote the density operator. The operators

$$
Y_{j_{\alpha}}^{\beta}=a_{j_{\alpha}}^{\dagger}\left(1-n_{j}\right) a_{j_{\beta}}
$$

are then obviously local projection operators, i.e. they satisfy

$$
Y_{j_{\alpha}}^{\beta} Y_{j_{\gamma}}^{\delta}=\delta_{\gamma}^{\beta} Y_{j_{\alpha}}^{\delta}
$$

They carry parity, induced by the anticommutation rule for the Fermi operators. Let $j \neq k$. Then $Y_{j}^{\beta}$ and $Y_{k_{\gamma}}^{\delta}$ anticommute, if both are build up of an odd number of Fermi operators, and commute in all other cases. This fact can be expressed as follows. Let $p(1)=0, p(2)=1$ and $p\left(Y_{j_{\alpha}}^{\beta}\right)=p(\alpha)+p(\beta)$. Then $Y_{j_{\alpha}}^{\beta}$ is odd (contains an odd number of Fermi operators), if $p\left(Y_{j_{\alpha}}^{\beta}\right)=1$, and even, if $p\left(Y_{j_{\alpha}}^{\beta}\right)=0$. The commutation rules for the projectors $Y_{j_{\alpha}}^{\beta}$ are thus

$$
Y_{j_{\alpha}}^{\beta} Y_{k_{\gamma}}^{\delta}=(-1)^{(p(\alpha)+p(\beta))(p(\gamma)+p(\delta))} Y_{k_{\gamma}}^{\delta} Y_{j}^{\beta},
$$

As a memorizing scheme for the projection operators let us combine them into the matrix $\left(Y_{j}\right)_{\beta}^{\alpha}=Y_{j}^{\beta}$,

$$
Y_{j}=\left(\begin{array}{cc}
1-n_{j} & c_{j} \\
c_{j}^{\dagger} & n_{j}
\end{array}\right) \quad
$$


(50) and (51) are representations of equations (14) and (16) in the particular case $m=n=1$. Since all our considerations in the previous sections entirely relied on eqs. (14) and (16), we may simply replace $e_{j_{\alpha}}^{\beta}$ by $Y_{j_{\alpha}}^{\beta}$ in eqs. (18), (27) and (40) to obtain fermionic representations of the permutation operator, the $L$-matrix and the Hamiltonian. The permutation operator (for $j \neq k$ ) becomes

$$
\begin{aligned}
P_{j k} & =Y_{j 1}^{1} Y_{k 1}^{1}+Y_{j_{2}}^{1} Y_{k 1}^{2}-Y_{j 1}^{2} Y_{k 2}^{1}-Y_{j_{2}}^{2} Y_{k 2}^{2} \\
& =1-\left(c_{j}^{\dagger}-c_{k}^{\dagger}\right)\left(c_{j}-c_{k}\right) .
\end{aligned}
$$

Fermionic representations compatible with arbitrary grading can be constructed by considering several species of fermions and graded products of projection operators. We shall explain this for the case of two species first. This is the most interesting case for applications, since we may interpret the two species as up- and down-spin electrons. We have to attach a spin index to the Fermi operators, $c_{j} \rightarrow c_{j \sigma}, \sigma=\uparrow, \downarrow,\left\{c_{j \sigma}, c_{k \tau}^{\dagger}\right\}=\delta_{j k} \delta_{\sigma \tau}$. Accordingly, there are two species of projection operators, $Y_{j_{\alpha}}^{\beta} \rightarrow Y_{j}^{\sigma \beta}$,

$$
Y_{j}^{\uparrow^{\beta}} Y_{j}^{\downarrow^{\delta}}=(-1)^{(p(\alpha)+p(\beta))(p(\gamma)+p(\delta))} Y_{j}^{\downarrow_{\gamma}^{\delta}} Y_{j \alpha}^{\uparrow \beta}
$$

Let us define projection operators for electrons by the tensor products

$$
Y_{j_{\alpha \gamma}}^{\beta \delta}=(-1)^{(p(\alpha)+p(\beta)) p(\gamma)} Y_{j}^{\downarrow_{\alpha}^{\beta}} Y_{j}^{\uparrow \delta}=\left(Y_{j}^{\downarrow} \otimes_{s} Y_{j}^{\uparrow}\right)_{\beta \delta}^{\alpha \gamma}
$$

Then

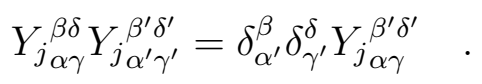

$Y_{j}^{\beta \delta}$ inherits the parity from $Y_{j}^{\downarrow^{\beta}}$ and $Y_{j}^{\uparrow^{\delta}}$. The number of Fermi operators contained in $Y_{j \alpha \gamma}^{\beta \delta}$ is the sum of the number of Fermi operators in $Y_{j}^{\downarrow^{\beta}}$ and $Y_{j}^{\uparrow \delta}$. Hence $p\left(Y_{j_{\alpha \gamma} \delta}^{\beta \delta}\right)=p\left(Y_{j}^{\downarrow^{\beta}}\right)+p\left(Y_{j}^{\uparrow^{\delta}}\right)=p(\alpha)+\ldots+p(\delta)$, and the analog of (51) holds for $Y_{j \alpha \gamma}^{\beta \delta}$, too. Again we present all projection operators in form of a matrix $\left(Y_{j}\right)_{\beta \delta}^{\alpha \gamma}=Y_{j}^{\beta \delta}$,

$$
Y_{j}=Y_{j}^{\downarrow} \otimes_{s} Y_{j}^{\uparrow}
$$




$$
=\left(\begin{array}{cccc}
\left(1-n_{j \downarrow}\right)\left(1-n_{j \uparrow}\right) & \left(1-n_{j \downarrow}\right) c_{j \uparrow} & c_{j \downarrow}\left(1-n_{j \uparrow}\right) & c_{j \downarrow} c_{j \uparrow} \\
\left(1-n_{j \downarrow}\right) c_{j \uparrow}^{\dagger} & \left(1-n_{j \downarrow}\right) n_{j \uparrow} & -c_{j \downarrow} c_{j \uparrow}^{\dagger} & -c_{j \downarrow} n_{j \uparrow} \\
c_{j \downarrow}^{\dagger}\left(1-n_{j \uparrow}\right) & c_{j \downarrow}^{\dagger} c_{j \uparrow} & n_{j \downarrow}\left(1-n_{j \uparrow}\right) & n_{j \downarrow} c_{j \uparrow} \\
-c_{j \downarrow}^{\dagger} c_{j \uparrow}^{\dagger} & -c_{j \downarrow}^{\dagger} n_{j \uparrow} & n_{j \downarrow} c_{j \uparrow}^{\dagger} & n_{j \downarrow} n_{j \uparrow}
\end{array}\right) .
$$

Here we used the standard ordering of matrix elements of tensor products, corresponding to a renumbering $(11) \rightarrow 1,(12) \rightarrow 2,(21) \rightarrow 3,(22) \rightarrow 4$. Within this convention $Y_{j \alpha}^{\beta \delta}$ is replaced by $Y_{j}^{\beta}, \alpha, \beta=1, \ldots, 4$, which then satisfies (50) and (51) with grading $p(1)=p(4)=0, p(2)=p(3)=1$.

The permutation operator of electrons turns out to be a product of permutation operators of up-and down-spin electrons,

$$
\begin{aligned}
P_{j k} & =(-1)^{p(\beta)+p(\delta)} Y_{j \alpha \gamma}^{\beta \delta} Y_{k \beta \delta}^{\alpha \gamma} \\
& =(-1)^{p(\beta)+p(\delta)+(p(\alpha)+p(\beta))(p(\gamma)+p(\delta))} Y_{j}^{\downarrow}{ }_{\alpha}^{\beta} Y_{j}^{\uparrow \delta} Y_{k \beta}^{\downarrow} Y_{k \delta}^{\alpha} \\
& =(-1)^{p(\beta)} Y_{j}^{\downarrow_{\alpha}^{\beta}} Y_{k \beta}^{\downarrow}{ }^{\alpha}(-1)^{p(\delta)} Y_{j}^{\uparrow \delta} Y_{k \delta}^{\uparrow \gamma}=P_{j k}^{\downarrow} P_{j k}^{\uparrow}=P_{j k}^{\uparrow} P_{j k}^{\downarrow}
\end{aligned}
$$

So far we have considered the case of spinless fermions with two-dimensional local space of states and grading $m=n=1$, and the case of electrons with four-dimensional space of states and grading $m=n=2$. There are four different possibilities to realize (14) and (16) in case of a three-dimensional local space of states, $m+n=3$. They can be obtained by deleting the $\alpha$ 's row and column of the matrix $Y_{j}$ in eq. (57), $\alpha=1,2,3,4$. (50) and (51) remain valid, since the operators $Y_{j}^{\beta}$ are projectors.

As an example let us consider the case where the fourth row and column of $Y_{j}$, eq. (57), are deleted. The local Hilbert space is then spanned by the three states $|0\rangle, c_{j \uparrow}^{\dagger}|0\rangle, c_{j \downarrow}^{\dagger}|0\rangle$, i.e. double occupancy is now excluded. The operator

$$
\sum_{\alpha=1}^{3} Y_{j_{\alpha}}^{\alpha}=1-Y_{j 4}^{4}=1-n_{j \uparrow} n_{j \downarrow}
$$

projects the local Hilbert space of electrons onto the space with no double occupancy. The global projection operator for a chain of $L$ sites is given by the product

$$
\Delta=\prod_{j=1}^{L}\left(1-n_{j \uparrow} n_{j \downarrow}\right)
$$


The permutation operator $P_{j k}$ is again given by eq. (18) with $Y_{j_{\alpha}}^{\beta}$ replacing $e_{j_{\alpha}}^{\beta}$. Summation is now over three values, $\alpha, \beta=1,2,3$, and the grading is $p(1)=0, p(2)=p(3)=1$. An elegant way of taking into account the simplifications arising from the restriction to the Hilbert space with with no double occupancy is to consider $P_{j k} \Delta$ instead of $P_{j k}$. Since $n_{j \uparrow} n_{j \downarrow} \Delta=0$, we obtain

$$
P_{j k} \Delta=\Delta\left(c_{j \sigma}^{\dagger} c_{k \sigma}+c_{k \sigma}^{\dagger} c_{j \sigma}\right) \Delta-2\left(S_{j}^{a} S_{k}^{a}-\frac{1}{4} n_{j} n_{k}\right) \Delta+\left(1-n_{j}-n_{k}\right) \Delta .
$$

Here we have introduced the electron density $n_{j}=n_{j \uparrow}+n_{j \downarrow}$ and the spin densities

$$
S_{j}^{a}=\frac{1}{2} \sigma_{\alpha \beta}^{a} c_{j \alpha}^{\dagger} c_{j \beta}
$$

The $\sigma^{a}, a=x, y, z$, are the Pauli matrices, and we identify 1 with $\uparrow$ an 2 with $\downarrow$ in the summation over $\alpha$ and $\beta$. The spin densities can alternatively be written as

$$
S_{j}^{a}=\frac{1}{2}\left(\sigma^{a}\right)_{\beta}^{\alpha} Y_{j_{\alpha+1}}^{\beta+1}
$$

Using

$$
\left(\sigma^{a} \otimes \sigma^{a}\right)_{\beta \delta}^{\alpha \gamma}=2 \delta_{\delta}^{\alpha} \delta_{\beta}^{\gamma}-\delta_{\beta}^{\alpha} \delta_{\delta}^{\gamma}
$$

we obtain

$$
\left(Y_{j_{1}}^{1} Y_{k 1}^{1}-\sum_{\alpha, \beta=2}^{3} Y_{j_{\alpha}}^{\beta} Y_{k \beta}^{\alpha}\right) \Delta=\left(-2\left(S_{j}^{a} S_{k}^{a}-\frac{1}{4} n_{j} n_{k}\right)+1-n_{j}-n_{k}\right) \Delta,
$$

which gives the second and third term on the right hand side of (61). Note that the permutation operator $P_{j k}$ in eq. (61) is no longer a product like in eq. (58).

It should be clear by now, how to generalize the above considerations to an arbitrary number of species of fermions. In the case of $N$ species we may define

$$
Y_{j_{1} \ldots \alpha_{N}}^{\beta_{1} \ldots \beta_{N}}=\left(Y_{j}^{N} \otimes_{s} \ldots \otimes_{s} Y_{j}^{1}\right)_{\beta_{1} \ldots \beta_{N}}^{\alpha_{1} \ldots \alpha_{N}}
$$

Then

$$
\begin{aligned}
& Y_{j_{1} \ldots \alpha_{N}}^{\beta_{1} \ldots \beta_{N}} Y_{j_{\gamma_{1} \ldots \gamma_{N}}}^{\delta_{1} \ldots \delta_{N}}=\delta_{\gamma_{1}}^{\beta_{1}} \ldots \delta_{\gamma_{N}}^{\beta_{N}} Y_{j_{\alpha_{1} \ldots \alpha_{N}}}^{\delta_{1} \ldots \delta_{N}}
\end{aligned}
$$

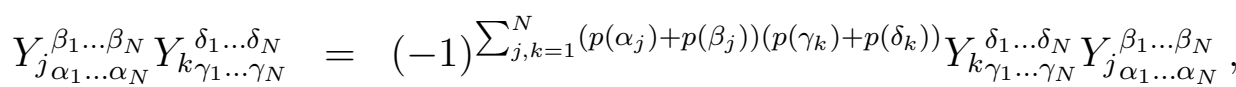


which can be shown by induction over the number of species. Here the grading is $m=n=2^{N-1}$. The most general case is obtained by deleting rows and columns from $Y_{j}$, eq. (66), in analogy to the example considered above.

Let us note that the operators $Y_{j_{\alpha}}^{\beta}$ for two species of fermions appear under the name Hubbard projection operators in the literature.

\section{Gauge transformations}

The canonical anticommutation relations (48) are invariant under local gauge transformations

$$
c_{j} \quad \rightarrow \quad \hat{c}_{j}=e^{\mathrm{i} \varphi_{j}} c_{j} \quad, \quad \varphi_{j} \in[0,2 \pi] .
$$

Thus all our formulae remain correct, if we replace $c_{j}$ by $\hat{c}_{j}$. The local gauge transformation induces a transformation of the matrix $Y_{j}$ of projection operators,

$$
Y_{j} \quad \rightarrow \quad \hat{Y}_{j}=e^{\mathrm{i} \varphi_{j} \sigma^{z} / 2} Y_{j} e^{-\mathrm{i} \varphi_{j} \sigma^{z} / 2}=e^{-\mathrm{i} \varphi_{j} n_{j}} Y_{j} e^{\mathrm{i} \varphi_{j} n_{j}} .
$$

Here $\sigma^{z}$ is a Pauli matrix. In the case of $N$ species of fermions there are $N$ gauge parameters $\varphi_{j}^{\sigma}, \sigma=1, \ldots, N$. Since $e^{\mathrm{i} \varphi_{j}^{\sigma} \sigma^{z} / 2}$ is diagonal, the form $(70)$ of the transformation rule for $Y_{j}$ carries over to the case of $N$ species. Let

$$
G_{j}=e^{\mathrm{i} \varphi_{j}^{N} \sigma^{z} / 2} \otimes \ldots \otimes e^{\mathrm{i} \varphi_{j}^{1} \sigma^{z} / 2}
$$

Then

$$
Y_{j} \quad \rightarrow \quad \hat{Y}_{j}=G_{j} Y_{j} G_{j}^{\dagger} .
$$

For the sake of simplicity we assume in the following that we are dealing with two species of fermions. Note, however, that the following considerations also apply in the most general case, where we would have to deal with a sub-matrix of $Y_{j}$, eq. (66). The $L$-matrix (27) behaves under gauge transformations as

$$
\hat{\mathcal{L}}_{j \beta}^{\alpha}=G_{j_{\alpha^{\prime}}}^{\dagger^{\alpha}}(-1)^{p\left(\alpha^{\prime}\right) p\left(\gamma^{\prime}\right)} \hat{R}_{j_{\beta^{\prime} \delta^{\prime}}}^{\alpha^{\prime} \gamma^{\prime}}(u, v) Y_{j_{\gamma^{\prime}}}^{\delta^{\prime}} G_{j \beta}^{\beta^{\prime}} .
$$

Here we defined

$$
\hat{R}_{j}(u, v)=\left(G_{j} \otimes G_{j}\right) R(u, v)\left(G_{j}^{\dagger} \otimes G_{j}^{\dagger}\right)
$$


Let us consider two implications of gauge invariance of the $R$-matrix.

(i) Global gauge transformations: Assume that $G_{j}=G, j=1, \ldots L$, and that $\hat{R}(u, v)=R(u, v)$, say, for arbitrary $\varphi^{\uparrow}, \varphi^{\downarrow}(\uparrow=1, \downarrow=2)$. Taking the derivative of (74) with respect to $\varphi^{\uparrow}$ at $\varphi^{\uparrow}=\varphi^{\downarrow}=0$ yields

$$
\left[R(u, v), I_{2} \otimes \sigma^{z} \otimes I_{4}+I_{4} \otimes I_{2} \otimes \sigma^{z}\right]=0 \quad .
$$

Since $I_{2} \otimes \sigma^{z} \otimes I_{4}$ and $I_{4} \otimes I_{2} \otimes \sigma^{z}$ are diagonal, we may replace $R(u, v)$ in this equation by $\tilde{R}(u, v)$. Then (44) is satisfied with $x=I_{2} \otimes \sigma^{z}$. Thus the transfer matrix commutes with

$$
X=\sum_{j=1}^{L}\left(Y_{j_{1}}^{1}-Y_{j_{2}}^{2}+Y_{j_{3}}^{3}-Y_{j_{4}}^{4}\right)=\sum_{j=1}^{L}\left(1-2 n_{j \uparrow}\right)
$$

which means that the number of up-spin electrons is conserved. A similar statement is easily verified for the number of down-spin electrons or, in the weaker case, when (74) is only satisfied for $\varphi^{\uparrow}=\varphi^{\downarrow}$, for the total number of electrons.

(ii) Local gauge transformations: Assume that $G_{j}=(G)^{j}$, where $G$ is a fixed matrix as in the example above, and that $R(u, v)$ is invariant under $G$. Then

$$
\hat{\mathcal{L}}_{j}(u, v)=\left(G^{\dagger}\right)^{j} \mathcal{L}_{j}(u, v)(G)^{j}
$$

and the transformed monodromy matrix becomes a simple expression in terms of the original $L$-matrices,

$$
\hat{\mathcal{T}}(u, v)=\left(G^{\dagger}\right)^{L} \mathcal{L}_{L}(u, v) G \mathcal{L}_{L-1}(u, v) G \ldots \mathcal{L}_{1}(u, v) G
$$

Transformations of this type can be used to introduce a phase factor into a typical nearest-neighbor hopping term as it appears in the Hubbard Hamiltonian. If the phase factor is just -1 , then the hopping term changes sign. The factor $\left(G^{\dagger}\right)^{L}$ generally modifies the transfer matrix $\operatorname{str}(\hat{\mathcal{T}}(u, v))$. It leads to a twist of the periodic boundary conditions. Note however, that it may happen for certain values of $L$, e.g. if $L$ is divisible by 2 or by 4 , that $\left(G^{\dagger}\right)^{L}=1$.

Gauge transformations of the type considered modify the shift operator. Using the right hand side of (770) in eq. (36) we can easily see that

$$
\hat{U} \quad \rightarrow \quad e^{\mathrm{i} L \varphi^{\sigma} n_{1 \sigma}-\mathrm{i} \varphi^{\sigma} \sum_{j=1}^{L} n_{j \sigma}} \hat{U}
$$


where summation over $\sigma=\uparrow, \downarrow$ is implied. The first term in the exponent of (79) is related to a twist of boundary conditions.

At this point will will not go into further detail, because we think that more detailed considerations are only sensible in the context of concrete models. Let us only remark that global symmetries of a given model may be affected by the exponent in eq. (79), even if there is no twist, i.e. even if the first term is equal to zero modulo $2 \pi$.

In the context of integrable fermionic models the term gauge transformation has, unfortunately, two different meanings, which should not be confused. Besides the meaning discussed above it is also used for transformations on the $R$-matrix of the form $R \rightarrow(G \otimes G) R\left(G^{-1} \otimes G^{-1}\right)$, where $G$ is not necessarily a diagonal matrix. Transformations of this form leave the YangBaxter equation (22) invariant.

\section{More examples}

To illustrate the formal discussions of the preceding sections let us further elaborate on the models with rational $R$-matrices (43). These models have been studied exhaustively in the literature. They appeared first as lattice gas models of Lai [12] and Sutherland [13] and were solved by coordinate Bethe ansatz. Later Kulish studied them within the framework of the graded quantum inverse scattering method and obtained spectrum and eigenstates by means of the nested algebraic Bethe ansatz [2]. Still Kulish did not write down any Hamiltonian density in terms of Fermi operators. This was first accomplished by Schlottmann for the gl(1|2) invariant case [14]. The Hamiltonian in fermionic representation is the Hamiltonian of the super symmetric $t$ - $J$-model. Schlottmann solved it again by means of the coordinate Bethe ansatz. However, he was not aware of the underlying algebraic structure. The underlying algebraic structure was successively unravelled by different authors [15, 16, 17] leading to a solution of the super symmetric $t$ - $J$-model by nested algebraic Bethe ansatz $\llbracket 17,18$. The gl $(2 \mid 2)$ invariant model with the Hamiltonian in fermionic representation was studied by Eßler et al. [19, 20].

Let us write down Hamiltonian, $R$-matrix, $L$-matrix and generators of symmetries for the $\mathrm{gl}(1 \mid 1)$ invariant case using the fermionic representation 
(52). The $R$-matrix follows from (43) with $p(1)=0, p(2)=1$,

$$
\check{R}(u, v)=\left(\begin{array}{rrrr}
1+u-v & & & \\
& 1 & u-v & \\
& u-v & 1 & \\
& & & 1-u+v
\end{array}\right)
$$

The $L$-matrix is obtained from (27),

$$
\begin{aligned}
\mathcal{L}_{j}(u, v) & =\left(\begin{array}{cc}
u-v+e_{j 1}^{1} & e_{j_{2}}^{1} \\
e_{j_{1}}^{2} & u-v-e_{j_{2}}^{2}
\end{array}\right) \\
& \rightarrow\left(\begin{array}{cc}
u-v+1-n_{j} & c_{j}^{\dagger} \\
c_{j} & u-v-n_{j}
\end{array}\right) .
\end{aligned}
$$

As Hamiltonian density we may choose

$$
\begin{aligned}
H_{j j+1} & =-P_{j j+1}=\left(c_{j}^{\dagger}-c_{j+1}^{\dagger}\right)\left(c_{j}-c_{j+1}\right)-1 \\
& =-\left(c_{j}^{\dagger} c_{j+1}+c_{j+1}^{\dagger} c_{j}\right)+n_{j}+n_{j+1}-1
\end{aligned}
$$

(cf (53)), which is the Hamiltonian density of a system of free fermions. The odd generators of symmetries are

$$
\begin{aligned}
& E_{2}^{1}=\sum_{j=1}^{L} e_{j_{2}}^{1} \rightarrow \sum_{j=1}^{L} c_{j}^{\dagger}, \\
& E_{1}^{2}=\sum_{j=1}^{L} e_{j_{1}^{2}} \rightarrow \sum_{j=1}^{L} c_{j} .
\end{aligned}
$$

From the even generators we can construct only one non-trivial combination,

$$
\frac{1}{2}\left(E_{2}^{2}-E_{1}^{1}+L\right) \quad \rightarrow \quad \hat{N}=\sum_{j=1}^{L} n_{j}
$$

Of course, being a model of free fermions, (83) is trivial in the sense that the corresponding Hamiltonian can be diagonalized by Fourier transform. We 
wrote down the above formulae in order to illustrate the general formalism. The reader can easily repeat all the steps for the case of the super symmetric $t$ - $J$-model, where the Hamiltonian density is given by $H_{j j+1}=-P_{j j+1}$ with $P_{j j+1}$ according to eq. (61).

Let us indicate the Hamiltonian density of the $\mathrm{gl}(2 \mid 2)$ invariant model [19, 20] using the fermionic representation (57). (43) and (40) imply that

$$
\begin{aligned}
H_{j j+1} & =-P_{j j+1}=-P_{j j+1}^{\uparrow} P_{j j+1}^{\downarrow} \\
& =-\left(1-\left(c_{j \uparrow}^{\dagger}-c_{j+1 \uparrow}^{\dagger}\right)\left(c_{j \uparrow}-c_{j+1 \uparrow}\right)\right)\left(1-\left(c_{j \downarrow}^{\dagger}-c_{j+1 \downarrow}^{\dagger}\right)\left(c_{j \downarrow}-c_{j+1 \downarrow}\right)\right) .
\end{aligned}
$$

Here the minus sign on the right hand side has been chosen in order to meet the usual conventions. If we open the brackets in (87), we obtain the rather voluminous expression $H_{j j+1}^{0}$ of Eßler, Korepin and Schoutens (cf e.g. eq. (8) in [19]), which we do not repeat here due to space limitations. Since $\left[P_{j j+1}, E_{\alpha}^{\beta}\right]=0, \alpha, \beta=1,2,3,4$, we may add, for instance, $U E_{4}^{4}, U$ real, to the Hamiltonian obtainable from (87) without spoiling integrability (see [19, 20]). This amounts to adding a Hubbard interaction $U n_{j \uparrow} n_{j \downarrow}$ to the Hamiltonian density. Note that the factorized form (87) of $H_{j j+1}$ appears to be new.

Here is a more recent example. Maassarani and Mathieu constructed an "su $(N)$ analogue" of the XX-model and found the corresponding $R$-matrix [8]. We will show that a certain fermionic representation of the "su(3) version" of this $R$-matrix generates the Hamiltonian of the Hubbard model at infinite coupling. Let us write the $R$-matrix of Maassarani and Mathieu in the form

$$
\begin{aligned}
& \check{R}(u, v \mid \delta)=\sin (u-v) \sum_{\alpha=2}^{N}\left(e^{\mathrm{i} \delta} e_{\alpha}^{1} \otimes e_{1}^{\alpha}+e^{-\mathrm{i} \delta} e_{1}^{\alpha} \otimes e_{\alpha}^{1}\right) \\
& \quad+\sum_{\alpha=2}^{N}\left(e_{1}^{1} \otimes e_{\alpha}^{\alpha}+e_{\alpha}^{\alpha} \otimes e_{1}^{1}\right)+\cos (u-v)\left(e_{1}^{1} \otimes e_{1}^{1}+\sum_{\alpha, \beta=2}^{N} e_{\alpha}^{\alpha} \otimes e_{\beta}^{\beta}\right) .
\end{aligned}
$$

Note that $\check{R}$ depends on an additional free parameter $\delta$. In order to meet our conventions we modified the original $R$-matrix of Maassarani and Mathieu by a gauge transformation $G, \check{R} \rightarrow(G \otimes G) \check{R}\left(G^{-1} \otimes G^{-1}\right), G e_{\alpha}^{\beta} G^{-1}=e_{(\alpha+1) \bmod N}^{(\beta+1) \bmod N}$. Clearly, $\check{R}$ is regular. Let us now restrict ourselves to the case $N=3$. Then $\check{R}$ is compatible with the grading $p(1)=0, p(2)=p(3)=1$. Let us consider 
the fermionic representation which is obtained from $Y_{j}$, eq. (57), by deleting the fourth row and column. Using (40) we obtain the Hamiltonian density

$$
H_{j j+1}=\sum_{\sigma=\uparrow, \downarrow}\left(e^{\mathrm{i} \delta} c_{j \sigma}^{\dagger} c_{j+1, \sigma}+e^{-\mathrm{i} \delta} c_{j+1, \sigma}^{\dagger} c_{j \sigma}\right)\left(1-n_{j,-\sigma}\right)\left(1-n_{j+1,-\sigma}\right) .
$$

We see that $\delta$ is connected to local gauge transformations. Setting $\delta=\pi$, $H_{j j+1}$ turns into the Hamiltonian density of the Hubbard model at infinite coupling, which is the same as the restricted hopping part of the super symmetric $t-J$-Hamiltonian. Hence the Hamiltonian can be written in the more familiar form

$$
H=-\sum_{j=1}^{L} \Delta\left(c_{j \sigma}^{\dagger} c_{j+1, \sigma}+c_{j+1, \sigma}^{\dagger} c_{j \sigma}\right) \Delta,
$$

where $\Delta$, eq. (60), is the projection operator which excludes double occupancy. Of course, (90) only makes sense, if the number of particles is less than $L$.

\section{Algebraic Bethe ansatz for the Hubbard model in the infinite coupling limit}

We can now apply the algebraic Bethe ansatz 21] to construct eigenvectors of the Hamiltonian (90). We shall regard equation (33) as a set of algebraic relations between the elements of the monodromy matrix $\mathcal{T}(u, w)$. Since $\mathcal{T}(u, w)$ is a function of $u-w$ in this case, we shall simply write $\mathcal{T}(u, w)=$ $\mathcal{T}(u-w)$ hereafter. Following Maassarani and Mathieu [8], we shall use the notation

$$
\mathcal{T}(u)=\left(\begin{array}{ccc}
S(u) & C_{1}(u) & C_{2}(u) \\
B_{1}(u) & t_{1}^{1}(u) & t_{2}^{1}(u) \\
B_{2}(u) & t_{1}^{2}(u) & t_{2}^{2}(u)
\end{array}\right)
$$

Let $|0\rangle$ denote the vacuum state defined by $c_{j}|0\rangle=0$. Using the explicit form of the $L$-matrix, which follows from (27), (88),

$$
\mathcal{L}_{j}(u)=\left(\begin{array}{ccc}
\cos u Y_{j_{1}}^{1}-\sin u\left(Y_{j_{2}}^{2}+Y_{j_{3}}^{3}\right) & Y_{j_{2}}^{1} & Y_{j_{3}}^{1} \\
Y_{j_{1}}^{2} & -\sin u Y_{j_{1}}^{1}-\cos u Y_{j_{2}}^{2} & -\cos u Y_{j_{3}}^{2} \\
Y_{j_{1}}^{3} & -\cos u Y_{j_{2}}^{3} & -\sin u Y_{j_{1}}^{1}-\cos u Y_{j_{3}}^{3}
\end{array}\right),
$$


we can easily see that

$$
\mathcal{T}(u)|0\rangle=\left(\begin{array}{ccc}
s(u)|0\rangle & * & * \\
0 & t(u)|0\rangle & 0 \\
0 & 0 & t(u)|0\rangle
\end{array}\right),
$$

where $s(u)=\cos ^{L}(u), t(u)=(-1)^{L} \sin ^{L}(u)$. This equation indicates that the vacuum $|0\rangle$ is an eigenstate of the transfer matrix. Following the procedure of the nested algebraic Bethe ansatz [2, 17], we assume the state

$$
\left|\lambda_{1}, \cdots, \lambda_{p}\right\rangle=F^{a_{1} \ldots a_{p}} C_{a_{1}}\left(\lambda_{1}\right) \ldots C_{a_{p}}\left(\lambda_{p}\right)|0\rangle
$$

to be an eigenstate of the transfer matrix. Here summation over $a_{j}=1,2$ is understood.

The commutation rules between the elements of the monodromy matrix can be extracted from (33),

$$
\begin{aligned}
C_{a}(u) C_{b}(v) & =C_{a}(v) C_{b}(u), \\
S(u) C_{a}(v) & =\cot (u-v) C_{a}(v) S(u)-\frac{C_{a}(u) S(v)}{\sin (u-v)}, \\
t_{a}^{a}(u) C_{b}(v) & =\cot (u-v) C_{a}(v) t_{b}^{a}(u)-\frac{C_{a}(u) t_{b}^{a}(v)}{\sin (u-v)} .
\end{aligned}
$$

Comparing these commutation rules with the ungraded case in [8], we notice that there appear extra minus signs when commuting $t_{a}^{a}(u)$ and $C_{b}(v)$. We use the above relations to calculate the action of the transfer matrix $\operatorname{str}(\mathcal{T}(u))$ on the state $\left|\lambda_{1}, \ldots, \lambda_{p}\right\rangle$. The resulting terms are classified as wanted terms and unwanted terms, respectively. The wanted terms are

$$
\begin{aligned}
& \prod_{j=1}^{p} \cot \left(u-\lambda_{j}\right)\left\{s(u)\left|\lambda_{1}, \ldots, \lambda_{p}\right\rangle\right. \\
& \left.\quad-t(u)\left(\tau^{(p)} F\right)^{a_{1} \ldots a_{p}} C_{a_{1}}\left(\lambda_{1}\right) \ldots C_{a_{p}}\left(\lambda_{p}\right)|0\rangle\right\} .
\end{aligned}
$$

Here $\tau^{(p)}$ is the shift operator on a $2^{L}$ dimensional auxiliary space, which may be realized as the space of states of a $p$-site spin one-half chain. Its matrix elements are given as

$$
\tau^{(p)}{ }_{a_{1} \ldots a_{p}}^{b_{1} \ldots b_{p}}=\delta_{a_{p}}^{b_{1}} \delta_{a_{1}}^{b_{2}} \ldots \delta_{a_{p-1}}^{b_{p}}
$$


The wanted terms are proportional to $\left|\lambda_{1}, \ldots, \lambda_{p}\right\rangle$, if $F$ is an eigenvector of the shift operator $\tau^{(p)}$ with eigenvalue $\Lambda^{(p)}$. On the other hand, in oder for the unwanted terms to cancel each other, we have the condition

$$
\tau^{(p)} F=(-1)^{L} \cot ^{L}\left(\lambda_{j}\right) F
$$

The spectrum of $\tau^{(p)}$ is fixed by the condition $\left(\tau^{(p)}\right)^{p}=1$. This condition implies that the spectrum consists of powers of the $p$-th root of unity (cf appendix $\mathrm{B}$ of [10]). The eigenvalue $\Lambda(u)$ of the transfer matrix $\operatorname{str}(\mathcal{T}(u))$ is thus given as

$$
\Lambda(u)=\left(s(u)-t(u) e^{\frac{\mathrm{i} 2 \pi l}{p}}\right) \prod_{j=1}^{p} \cot \left(u-\lambda_{j}\right),
$$

where $\lambda_{j}, j=1, \ldots, p$, is a solution of the Bethe ansatz equations

$$
(-1)^{L} \cot ^{L}\left(\lambda_{j}\right)=e^{\frac{\mathrm{i} 2 \pi l}{p}} \quad, \quad l=0, \ldots, p-1 .
$$

The only remaining problem is the explicit construction of eigenvectors of the shift operator $\tau^{(p)}$. This is, of course, a highly degenerate problem. We may use any integrable spin one-half chain with regular $R$-matrix to solve this problem. A particularly simple choice is the XX spin chain. Its $R$-matrix $\check{R}$ is given by eq. (88) with $N=2$ and $\delta=0$. The $L$-matrix in (non-graded) spin representation is

$$
L_{j}(u)=\left(\begin{array}{cc}
\cos (u) e_{j_{1}}^{1}+\sin (u) e_{j_{2}}^{2} & e_{j_{2}}^{1} \\
e_{j_{1}}^{2} & \sin (u) e_{j_{1}}^{1}+\cos (u) e_{j_{2}}^{2}
\end{array}\right)
$$

Let us use the notation

$$
T(u)=\left(\begin{array}{ll}
A(u) & B(u) \\
C(u) & D(u)
\end{array}\right)
$$

for the monodromy matrix $T(u)=L_{p}(u) \ldots L_{1}(u)$. With the choice $\left.\mid 0\right)=$ $\left(\begin{array}{l}1 \\ 0\end{array}\right)^{\otimes p}$ of auxiliary vacuum, we obtain

$$
T(u) \mid 0)=\left(\begin{array}{cc}
\left.\cos ^{p}(u) \mid 0\right) & * \\
0 & \left.\sin ^{p}(u) \mid 0\right)
\end{array}\right)
$$


which shows that the auxiliary vacuum is an eigenstate of the transfer matrix $\operatorname{tr}(T(u))$. To construct the eigenstates of $\operatorname{tr}(T(u))$ we need the commutation relations

$$
\begin{aligned}
{[B(u), B(v)] } & =0 \\
A(u) B(v) & =-\cot (u-v) B(v) A(u)+\frac{B(u) A(v)}{\sin (u-v)}, \\
D(u) B(v) & =\cot (u-v) B(v) D(u)-\frac{B(u) D(v)}{\sin (u-v)},
\end{aligned}
$$

which are part of the Yang-Baxter algebra (33). Proceeding as above, we consider the action of the transfer matrix $\operatorname{tr}(T(u))=A(u)+D(u)$ on the state

$$
\left.\left.\mid \mu_{1}, \ldots, \mu_{s}\right)=B\left(\mu_{1}\right) \ldots B\left(\mu_{s}\right) \mid 0\right) \quad .
$$

It turns out that $\left.\mid \mu_{1}, \ldots, \mu_{s}\right)$ is an eigenstate of $\operatorname{tr}(T(u))$ with eigenvalue

$$
\Lambda^{(p)}(u)=\left\{(-1)^{s} \cos ^{p}(u)+\sin ^{p}(u)\right\} \prod_{j=1}^{s} \cot \left(u-\mu_{j}\right)
$$

if the parameters $\mu_{j}, j=1, \ldots, s$, satisfy the equation

$$
\cot ^{p}\left(\mu_{j}\right)=(-1)^{s+1}
$$

Eq. (110) implies in particular that

$$
\Lambda^{(p)}(0)=\prod_{j=1}^{s} \cot \left(\mu_{j}\right)
$$

In order to introduce a convenient basis let us use the operators $e_{j_{a}}^{1} \cdot e_{j_{a}}^{1}$ applied to the auxiliary vacuum $\mid 0$ ) places an $\uparrow$-spin at site $j$, if $a=1$, and a $\downarrow$-spin, if $a=2$. The states $\left.e_{1_{1}}^{1} \ldots e_{p_{a_{p}}}^{1} \mid 0\right)$ form an orthonormal basis of our auxiliary Hilbert space, since $e_{j_{1}}^{b} e_{j_{a}}^{1}=\delta_{a}^{b} e_{j_{1}}^{1}$ and $\left.\left.e_{j_{1}}{ }^{1} \mid 0\right)=\mid 0\right)$. The components of the shift operator $\hat{U}=\operatorname{tr}(T(0))$ with respects to this basis are

$$
\begin{aligned}
& \left(0\left|e_{p 1}^{b_{p}} \ldots e_{11}^{b_{1}} \hat{U} e_{1_{a_{1}}}^{1} \ldots e_{p_{a_{p}}}^{1}\right| 0\right)= \\
& \left(0 \mid e_{p_{1}}^{b_{p}} \ldots e_{11}^{b_{1}} e_{2}^{1} \ldots e_{p_{a_{p-1}}}^{1} e_{\left.1_{a_{p}} \mid 0\right)=\delta_{a_{p}}^{1} \delta_{a_{1}}^{b_{1}} \ldots \delta_{a_{p-1}}^{b_{p}},},\right.
\end{aligned}
$$


such that we can identify $\tau^{(p)}$ with $\hat{U}$. Let $\left.F=\mid \mu_{1}, \ldots, \mu_{s}\right)$. It follows from eq. (112) that

$$
\tau^{(p)} F=\prod_{j=1}^{s} \cot \left(\mu_{j}\right) F
$$

Now eq. (111) implies that

$$
\left(\prod_{j=1}^{s} \cot \left(\mu_{j}\right)\right)^{p}=(-1)^{s(s+1)}=1,
$$

and we have verified that the eigenvalues of $\tau^{(p)}$ are indeed powers of the $p$ th root of unity. Note the the components of $F$ with respect to our basis, which enter the definition (94) of $\left|\lambda_{1}, \ldots, \lambda_{p}\right\rangle$ can be written as

$$
F^{a_{1} \ldots a_{p}}=\left(0\left|e_{p 1}^{a_{p}} \ldots e_{1}^{a_{1}} B\left(\mu_{1}\right) \ldots B\left(\mu_{s}\right)\right| 0\right) \quad .
$$

We can use the symmetries of the monodromy matrices $T(u)$ and $\mathcal{T}(u)$ to deduce restrictions on the numbers $p$ and $s$. Eq. (44) implies that the $R$-matrix of the XX chain is invariant under $\sigma^{z},\left[R, \sigma^{z} \otimes I_{2}+I_{2} \otimes \sigma^{z}\right]=0$. Let

$$
\Sigma^{z}=\sum_{j=1}^{p} \sigma_{j}^{z}
$$

This is twice the operator of the $z$-component of the total spin. We infer from (46) that $\left[T(u), \sigma^{z}\right]=\left[\Sigma^{z}, T(u)\right] . B(u)$, in particular, commutes with $\Sigma^{z}$ as

$$
\Sigma^{z} B(u)=B(u)\left(\Sigma^{z}-2\right)
$$

Thus

$$
\left.\left.\frac{1}{2} \Sigma^{z} \mid \mu_{1}, \ldots, \mu_{s}\right)=\frac{1}{2}(p-2 s) \mid \mu_{1}, \ldots, \mu_{s}\right),
$$

$\left.\mid \mu_{1}, \ldots, \mu_{s}\right)$ is an eigenvector of the $z$-component of the operator of total spin with eigenvalue $\frac{1}{2}(p-2 s)$. This eigenvalue cannot be smaller than $-\frac{1}{2} p$. Therefore $s \leq p$.

Using once more eq. (44) we find that the $R$-matrix of the Hubbard model in the infinite coupling limit is invariant under the even generators of $g l(1 \mid 2)$, $e_{1}^{1}, e_{2}^{2}, e_{3}^{3}, e_{3}^{2}, e_{2}^{3}$. They span the Lie algebra $\operatorname{gl}(1) \oplus \operatorname{gl}(2)$. The matrix $e_{2}^{2}+e_{3}^{3}$ generates the symmetry operator

$$
\hat{N}=\sum_{j=1}^{L}\left(Y_{j 2}^{2}+Y_{j 3}^{3}\right)=\sum_{j=1}^{L}\left(n_{j \uparrow}+n_{j \downarrow}-2 n_{j \uparrow} n_{j \downarrow}\right)
$$


This is the particle number operator on our restricted Hilbert space, where double occupancy of sites is forbidden. Eq. (46) implies that $[\hat{N}, \mathcal{T}(u)]=$ $\left[\mathcal{T}(u), e_{2}^{2}+e_{3}^{3}\right]$ and, in particular, that

$$
\hat{N} C_{a}(u)=C_{a}(u)(\hat{N}+1)
$$

We conclude that

$$
\hat{N}\left|\lambda_{1}, \ldots, \lambda_{p}\right\rangle=p\left|\lambda_{1}, \ldots, \lambda_{p}\right\rangle
$$

The state $\left|\lambda_{1}, \ldots, \lambda_{p}\right\rangle$ is constructed by acting on the vacuum with operators, which leave the Hilbert space with no double occupancy invariant. Therefore it can contain at most $L$ particles, and $p \leq L$.

Let us perform a naive counting of states. We will start with the auxiliary spin states $\left.\mid \mu_{1}, \ldots, \mu_{s}\right)$. Eq. (111) has $p$ solutions for every $j=1, \ldots s$. The equivalence of XX chain and a chain of spinless fermions suggests that all $\mu_{j}$ have to be different. States $\left.\mid \mu_{1}, \ldots, \mu_{s}\right)$, which merely differ by the order of the $\mu_{j}$, are identical due to (106). It follows that we can order the $\mu_{j}$ as $\mu_{1}<\ldots<\mu_{s}$. The number of solutions of (111) which satisfy this ordering is $\sum_{s=0}^{p}\left(\begin{array}{l}p \\ s\end{array}\right)=2^{p}$. Let us assume that the $\lambda_{j}$, too, are pairwise distinct. Then we can order them as $\lambda_{1}<\ldots<\lambda_{p}$, because of (95). The total number of states satisfying the above restrictions is $\sum_{p=0}^{L}\left(\begin{array}{l}L \\ p\end{array}\right) 2^{p}=3^{L}$, which is the dimension of our Hilbert space. This strongly suggests that our algebraic Bethe ansatz solution provides us with a complete set of states. An actual proof would require to calculate all scalar products of the form $\left\langle\lambda_{1}, \ldots, \lambda_{p} \mid \lambda_{1}^{\prime}, \ldots, \lambda_{p}^{\prime}\right\rangle$, which is feasible, yet beyond the scope of this article.

We want to point out, that the states $\left|\lambda_{1}, \ldots, \lambda_{p}\right\rangle$ are eigenstates of the $z$-component of the total spin operator. The proof goes as follows. The $R$ matrix of the Hubbard model in the infinite coupling limit is invariant under $\frac{1}{2}\left(e_{2}^{2}-e_{3}^{3}\right)$. Now

$$
S^{z}=\frac{1}{2} \sum_{j=1}^{L}\left(n_{j \uparrow}-n_{j \downarrow}\right)=\frac{1}{2} \sum_{j=1}^{L}\left(Y_{j_{2}}^{2}-Y_{j_{3}}^{3}\right),
$$

and eq. (46) implies that $\left[S^{z}, \mathcal{T}(u)\right]=\frac{1}{2}\left[\mathcal{T}(u), e_{2}^{2}-e_{3}^{3}\right]$, or, for the elements $C_{a}(u)$ of the monodromy matrix,

$$
\left[S^{z}, C_{a}(u)\right]=\frac{1}{2}\left(\sigma^{z}\right)_{a}^{b} C_{b}(u) .
$$


It follows that

$$
\begin{aligned}
S^{z}\left|\lambda_{1}, \ldots, \lambda_{p}\right\rangle & =\frac{1}{2}\left(\Sigma^{z} F\right)^{a_{1} \ldots a_{p}} C_{a_{1}}\left(\lambda_{1}\right) \ldots C_{a_{p}}\left(\lambda_{p}\right)|0\rangle \\
& =\frac{1}{2}(p-2 s)\left|\lambda_{1}, \ldots, \lambda_{p}\right\rangle .
\end{aligned}
$$

Let us compare our results with the recent coordinate Bethe ansatz solution of the model by Izergin et al. [22]. The one-particle algebraic Bethe ansatz states are easily shown to be of the form

$$
C_{a}(\lambda)|0\rangle \propto \sum_{j=1}^{L}(-\tan (\lambda))^{j} c_{j, a}^{\dagger}|0\rangle \quad,
$$

where $c_{j a}=c_{j \uparrow}$, if $a=1$, and $c_{j a}=c_{j \downarrow}$, if $a=2$. From this equation we identify the quasimomenta $k(\lambda)$ of one-particle states as

$$
e^{\mathrm{i} k(\lambda)}=-\tan (\lambda)
$$

Let us perform a similar reparametrization for the auxiliary spin problem,

$$
e^{\mathrm{i} q(\mu)}=\tan (\mu)
$$

Then, using (115), we can rewrite the Bethe ansatz equations (102) and (111) as

$$
\begin{gathered}
e^{\mathrm{i} k_{j} L}=e^{\mathrm{i} \sum_{k=1}^{s} q_{k}} \quad, \quad j=1, \ldots, p, \\
e^{\mathrm{i} q_{k} p}=(-1)^{s+1}, \quad k=1, \ldots, s,
\end{gathered}
$$

where $k_{j}=k\left(\lambda_{j}\right), q_{k}=q\left(\mu_{k}\right)$. These equations agree with the Bethe ansatz equations obtained in 22.

Eq. (37) implies that the energy $E$ of a state $\left|\lambda_{1}, \ldots \lambda_{p}\right\rangle$ is

$$
\begin{aligned}
E & =\left.\partial_{u} \ln (\Lambda(u))\right|_{u=0}=\sum_{j=1}^{p}\left(\tan \left(\lambda_{j}\right)+\cot \left(\lambda_{j}\right)\right) \\
& =-2 \sum_{j=1}^{p} \cos \left(k_{j}\right) .
\end{aligned}
$$

Eqs. (122) and (125) show that the states $\left|\lambda_{1}, \ldots \lambda_{p}\right\rangle$ are also eigenstates of the particle number operator $\hat{N}$ and the $z$-component of the total spin $S^{z}$. We 
may thus add a chemical potential and a magnetic field to our Hamiltonian (90), $H \rightarrow H_{\mu, B}$,

$$
H_{\mu, B}=\sum_{j=1}^{L}\left\{-\Delta\left(c_{j \sigma}^{\dagger} c_{j+1, \sigma}+c_{j+1, \sigma}^{\dagger} c_{j \sigma}\right) \Delta-\mu\left(n_{j \uparrow}+n_{j \downarrow}\right)+B\left(n_{j \uparrow}-n_{j \downarrow}\right)\right\} .
$$

Then $H_{\mu, B}\left|\lambda_{1}, \ldots, \lambda_{p}\right\rangle=E_{\mu, B}\left|\lambda_{1}, \ldots, \lambda_{p}\right\rangle$, where

$$
E_{\mu, B}=-2 \sum_{j=1}^{p} \cos \left(k_{j}\right)-\mu p+B(p-2 s)
$$

This means that the algebraic Bethe ansatz states $\left|\lambda_{1}, \ldots, \lambda_{p}\right\rangle$ can be used to calculate form factors and correlation functions in the grand canonical ensemble (cf [22]).

\section{Yangian symmetry of the Hubbard model in the infinite coupling limit}

The Hubbard Hamiltonian on the infinite interval is invariant under the direct sum of two $\mathrm{su}(2)$ Yangian quantum groups [23, 24]. We would like to point out here, that one of these Yangian symmetries survives the infinite coupling limit. First of all, let us recall [25] that the su(2) Yangian is a Hopf algebra which is spanned by two triples of generators $I^{a}, J^{a}, a=x, y, z$, satisfying the relations

$$
\begin{aligned}
{\left[I^{a}, I^{b}\right] } & =c_{a b c} I^{c}, \\
{\left[I^{a}, J^{b}\right]=} & c_{a b c} J^{c}, \\
{\left[\left[J^{a}, J^{b}\right],\left[I^{c}, J^{d}\right]\right]+} & {\left[\left[J^{c}, J^{d}\right],\left[I^{a}, J^{b}\right]\right]=} \\
& -4\left(a_{a b e f g h} c_{c d e}+a_{c d e f g h} c_{a b e}\right)\left\{I^{f}, I^{g}, J^{h}\right\} .
\end{aligned}
$$

Here $c_{a b c}=\mathrm{i} \varepsilon^{a b c}$ is the antisymmetric tensor of structure constants of $\mathrm{su}(2)$, and $a_{a b c d e f}=c_{a d g} c_{b e h} c_{c f i} c_{g h i}$. The bracket \{\} in (136) denotes the symmetrized product

$$
\left\{x_{1}, x_{2}, x_{3}\right\}=\frac{1}{6} \sum_{i \neq j \neq k \neq i} x_{i} x_{j} x_{k}
$$


Being a Hopf algebra $\mathrm{Y}(\mathrm{su}(2))$ carries an outer structure (co-multiplication, antipode, co-unit), which guarantees that $\mathrm{Y}(\mathrm{su}(2))$ has a rich representation theory [26].

As a corollary of a theorem proven in [27] it follows that

$$
\begin{aligned}
I^{a} & =\sum_{j} S_{j}^{a}, \\
J^{a} & =\sum_{j \neq k} \operatorname{sign}(j-k) \varepsilon^{a b c} S_{j}^{b} S_{k}^{c}
\end{aligned}
$$

form a representation of $\mathrm{Y}(\mathrm{su}(2))$. The $S_{j}^{a}$ in this equation are spin density operators in fermionic representation (62). $I^{a}$ obviously commutes with the Hubbard Hamiltonian in the infinite coupling limit (90). We will give a simple proof [28] that $J^{a}$, too, commutes with the Hamiltonian (90), if we replace the summation in (90) by a summation over all integers. Let $S_{j k}^{a}=\frac{1}{2} \sigma_{\alpha \beta}^{a} c_{j \alpha}^{\dagger} c_{k \beta}$, and define

$$
K^{a}=2 \mathrm{i} \sum_{j}\left(S_{j, j+1}^{a}-S_{j+1, j}^{a}\right)
$$

It was shown in [23, 27] that the Hubbard Hamiltonian $H_{H}$,

$$
\begin{aligned}
H_{H} & =T+U D, \\
T & =-\sum_{j}\left(c_{j \sigma}^{\dagger} c_{j+1 \sigma}+c_{j+1 \sigma}^{\dagger} c_{j \sigma}\right), \\
D & =\sum_{j} n_{j \uparrow} n_{j \downarrow},
\end{aligned}
$$

commutes with

$$
J_{U}^{a}=J^{a}+U^{-1} K^{a}
$$

and that $I^{a}$ and $J_{U}^{a}$ form a representation of the $\mathrm{Y}(\mathrm{su}(2))$ Yangian. Since $J_{U}^{a}$ commutes with $H_{H}$ for all real $U$, we obtain the identity $\left[T, J^{a}\right]=\left[K^{a}, D\right]$. Now $D \Delta=\Delta D=0$, and $\left[\Delta, J^{a}\right]=0$, since $\left[\Delta, S_{j}^{a}\right]=0$. It follows that

$$
\left[H, J^{a}\right]=\left[\Delta T \Delta, J^{a}\right]=\Delta\left[T, J^{a}\right] \Delta=\Delta\left[K^{a}, D\right] \Delta=0 .
$$

Let us remark that a more systematic way to explore the Yangian symmetry of the model would be to apply the approach developed in [29, 24, 30], where the Yang-Baxter algebra was investigated in the thermodynamic limit. This approach would also provide us with the action of the Yangian on eigenstates. 


\section{Conclusions}

We would like to stress that our approach is widely applicable. Given a solution $R(u, v)$ of the Yang-Baxter equation (22), it consists of the following steps.

(i) Use eq. (26) to choose a grading which is compatible with the $R$-matrix. In general, this choice is not unique. A given $R$-matrix may be compatible with different gradings.

(ii) For a given grading choose a fermionic representation. Start with a tensor product representation (66) of projection operators $Y_{j_{\alpha}}^{\beta}$ of sufficiently high dimension and adjust it to the grading by deleting rows and columns of $Y_{j_{\alpha}}^{\beta}$. Again the choice is not unique.

(iii) Write down the Hamiltonian (40) and replace the graded spin operators $e_{j_{\alpha}}^{\beta}$ by fermionic projection operators $Y_{j_{\alpha}}^{\beta}$. This Hamiltonian is integrable by construction. There may be different methods to diagonalize it.

(iv) The Hamiltonian can be diagonalized by (nested) coordinate Bethe ansatz.

(v) It may be possible to diagonalize the Hamiltonian algebraically. In this case the $L$-matrix is obtained from eq. (27), and the graded YangBaxter algebra is given by (30). Note however, that there is no general recipe for an algebraic Bethe ansatz. Depending on the structure of the $R$-matrix and of the monodromy matrix an algebraic Bethe ansatz may be a difficult task (cf e.g. [31, 32]), if it is possible at all.

Our approach circumvents certain difficulties connected with the JordanWigner transformation, which, in certain cases, may be alternatively used to connect a spin representation of an integrable system with a fermionic representation. In our approach there is no twisting of the boundary conditions, and symmetries are directly obtainable via eq. (44). Our approach works for higher $\mathrm{su}(N)$ spins where no Jordan-Wigner transformation is known.

The main example to illustrate our ideas was the Hubbard model in the infinite coupling limit. An interesting lesson to learn from this example is that the Hubbard model in the infinite coupling limit is equivalent to a su(3)spin generalization of the XX spin chain. 


\section{Acknowledgements}

F. G. acknowledges partial financial support by the Deutsche Forschungsgemeinschaft under grant number Go 825/2-1.

\section{References}

[1] P. P. Kulish and E. K. Sklyanin, J. Soviet Math. 19, 1596 (1982).

[2] P. P. Kulish, J. Soviet Math. 35, 2648 (1985).

[3] A. J. Bracken, M. D. Gould, Y.-Z. Zhang, and G. W. Delius, J. Phys. A 27, 6551 (1994).

[4] G. W. Delius, M. D. Gould, J. R. Links, and Y.-Z. Zhang, Int. J. Mod. Phys. A 10, 3259 (1995).

[5] Z. Maassarani, J. Phys. A 28, 1305 (1995).

[6] B. S. Shastry, J. Stat. Phys. 50, 57 (1988).

[7] E. Olmedilla, M. Wadati, and Y. Akutsu, J. Phys. Soc. Jpn. 56, 2298 (1987).

[8] Z. Maassarani and P. Mathieu, Nucl. Phys. B 517, 395 (1998).

[9] M. Lüscher, Nucl. Phys. B 117, 475 (1976).

[10] F. Göhmann and S. Murakami, J. Phys. A 30, 5269 (1997).

[11] M. Shiroishi, H. Ujino, and M. Wadati, J. Phys. A 31, 2341 (1998).

[12] C. K. Lai, J. Math. Phys. 15, 1675 (1974).

[13] B. Sutherland, Phys. Rev. B 12, 3795 (1975).

[14] P. Schlottmann, Phys. Rev. B 36, 5177 (1987).

[15] P. A. Bares and G.Blatter, Phys. Rev. Lett. 64, 2567 (1990).

[16] S. Sarkar, J. Phys. A 24, 1137 (1991). 
[17] F. H. L. Eßler and V. E. Korepin, Phys. Rev. B 46, 9147 (1992).

[18] A. Foerster and M. Karowski, Nucl. Phys. B 396, 611 (1993).

[19] F. H. L. Eßler, V. E. Korepin, and K. Schoutens, Phys. Rev. Lett. 68, 2960 (1992).

[20] F. H. L. Eßler, V. E. Korepin, and K. Schoutens, Int. J. Mod. Phys. B 8, 3205 (1994).

[21] V. E. Korepin, N. M. Bogoliubov, and A. G. Izergin, Quantum Inverse Scattering Method and Correlation Functions, Cambridge University Press, (1993).

[22] A. G. Izergin, A. G. Pronko, and N. I. Abarenkova. Temperature correlators in the one-dimensional Hubbard model in the strong coupling limit. PDMI preprint 5/1998, hep-th/9801167, (1998).

[23] D. B. Uglov and V. E. Korepin, Phys. Lett. A 190, 238 (1994).

[24] S. Murakami and F. Göhmann, Phys. Lett. A 227, 216 (1997).

[25] V. G. Drinfel'd, Soviet Math. Dokl. 32, 254 (1985).

[26] V. Chari and A. Pressley, L'Enseignement Mathématique 36, 267 (1990).

[27] F. Göhmann and V. Inozemtsev, Phys. Lett. A 214, 161 (1996).

[28] V. Inozemtsev, private communication, (1995).

[29] S. Murakami and M. Wadati, J. Phys. A 29, 7903 (1996).

[30] S. Murakami and F. Göhmann, Nucl. Phys. B 512, 637 (1998).

[31] V. O. Tarasov, Theor. Math. Phys. 76, 793 (1988).

[32] P. B. Ramos and M. J. Martins, J. Phys. A 30, L195 (1997). 